\title{
Performance Evaluation of OFDM System using Pseudo-Pilots with Particle Swarm \& Moth Flame (Hybrid) Optimization
}

\author{
Mamta Arora, Paras Chawla
}

\begin{abstract}
This paper is focused on the advanced signal processing techniques for the multi-carrier modulation especially on the orthogonal frequency division multiplexing (OFDM). OFDM has high data rate and robust against the frequency selective multi-path channels. Channel estimation is crucial for the receiver design in coherent detection. In this paper we are investigating and examining the pilot aided system for channel estimation and its special effects on the performance of the OFDM based system. In this paper new technique is proposed which make use of conventional Least Square (LS) and Recursive Least Square (RLS) hybridization techniques for the channel estimation afterwards we apply Particle Swarm Optimization (PSO) and Hybrid PSO (PSO+ Moth Flame Optimization(MFO)) optimization for obtaining more optimize techniques. In the proposed system, to estimate channel impulse response we are using pseudo-pilots instead to pilots as it is useful to overcome the pilot overhead and decrease the complexity by using pseudo random symbol (PRS). The performance of the proposed techniques and the weighted scheme are compared and verified using computerized simulation carried out using Matrix Laboratory (MATLAB) software. It is demonstrated on the basis of graph between BER and signal to Noise Ratio (Eb/NO), that the proposed technique reduces the execution time and increase the transmission rate with low or zero overhead.
\end{abstract}

Index Terms: OFDM, PSO, MFO, LS, RLS, Channel estimation.

\section{INTRODUCTION}

modulation in the method of orthogonal frequency division multiplexing (OFDM) has be successful in wireless communications due to its extraordinary rate transmission ability with great band width efficiency and its sturdiness with regard to multi-path fading and long delay [1, 2, 3, 4].In an OFDM system, a (large) number of orthogonal, overlying, narrowband sub-channels orsub-carriers, communicated in equivalent, split the available transmission bandwidth. The addition of an extra guard interval amid successive OFDM symbols, realized by zero-padding, can reduce the effects of ISI even more and preserve orthogonality of the sub-carriers. Additional reason for OFDM as a specific kind of multicarrier modulation has been widely used for high-data-rate transmissions in delay-dispersive environments is that it has

Revised Manuscript Received on July 02, 2019

Mamta Arora ECED, Chandigarh University, Gharuan, Mohali, India

Paras Chawla ECED, Chandigarh University, Gharuan, Mohali, India significant advantages over time-domain equalization. Precise channel estimation can be used in OFDM systems to increase their performance by permitting for coherent demodulation. Retrieval of the transmitted data needs to estimate the Channel State Information (CSI). At the receiver side, CSI is found by employing different estimation techniques. In OFDM system we supplement pilots with payload symbol in direction to obtain channel estimation and for synchronization purpose. As the amount of pilot transmitted each independently pilot burst, disturbing the array of state in time domain and this leads to overhead problem in OFDM system [5]. It is also problematic to select the data for pilot i.e. plan of pilot data and plan of estimator with low randomness and great channel following capacity. Pilot overhead limits the performance, so it is essential to overcome the load in system due to pilot and understand the maximum pilot at receiver. In direction to increase the system performance, reduce overhead issue and Bit Error Rate (BER) performance of the OFDM system through Least Square (LS) estimator, it is necessary to control this problem. Overhead is leading concern in pilot aided system that confines its performance. To control overhead and advance performance we will employ Pseudo-Pilot instead of pilots. Pseudo-pilot condensed or even provides zero overhead with identical performance as conventional pilot system. Pseudo-pilot with hybrid of LS and RLS estimator is used as it mitigate overhead and improve OFDM performance with low complexity, delay time and high transmission rate. To optimize the whole process we are using particle swarm optimization (PSO) and moth flame optimization (MFO).

\section{PROPOSED SYSTEM MODEL}

Figure: 1 presents a typical block diagram OFDM System with Pseudo-pilot and Hybrid channel estimation Technique with PSO and MFO optimization techniques.To astounded the overhead in OFDM system we requisite a new pilot insertion approach which overwhelmed the overhead and follow highly predictable estimator. Pseudo-pilot is a novel paradigm of channel estimation in which we use the non-pilot assisted approach for channel estimation.The symbols transmitted through the uncertainty, serves as pseudo-pilots for the receiver to perform channel estimation. Our main idea is to generate the pseudo random symbol (PRS) by using interleavers where we transfer the numbers of payload symbols by employing the banks of interleavers at the transmitter side. Basic idea is to introduce pseudo random symbol interleavers also known as interleaver bank. At interleaver sample of signal is consider after 
shuffling of sample signal. The interleaver signal is rearrange in such a way that one of the arrangement of system is consider as reference and selected by selector which match one of the sub-lock before transmission of the signal. Interleaver rearranges the payload symbols block in numbers of ways so that at least one of the arrangement is contains a sub-block. Symbols within the sub-block are known as pseudo-pilot and these symbols are already known at receiver.

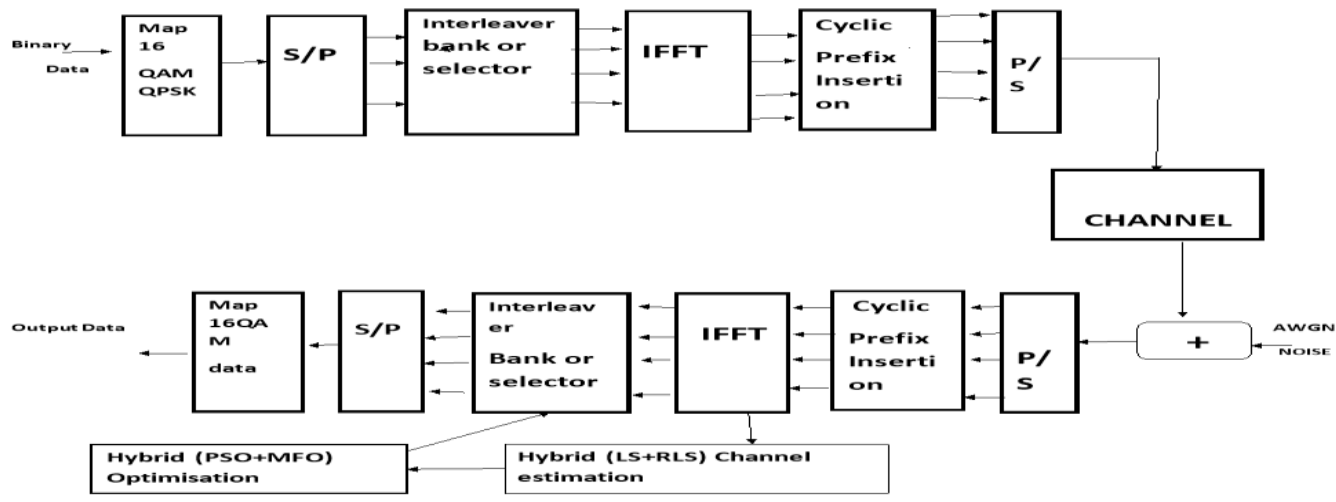

Figure 1: The proposed system Model

Now these pseudo-pilot generated by interleaver is used for the channel estimation process in OFDM system. In the conventional OFDM system pilot are transmitted with the payload symbols which used to find channel impulse response but here bank of interleavers used to generate PRS those are served for channel estimation process. In the OFDM system there are many estimator used for channel estimation purpose we applied the Hybrid (LS + RLS) estimation approach to recover the original transmitted information. To improve the results we are going to use Particle swarm optimization (PSO) and moth flame optimization (MFO) [11-12] technique to optimize the obtained result after the hybrid estimation.

\section{PARTICLE SWARM OPTIMIZATION}

The Particle Swarm Optimization is one of thevital stochastic technique which works on swarm of practices and movement of swarms in multi-dimensional exploration to find out optimum solution. PSO has very simple implementation andfast convergence ability. In this software agents are particles which give us potential solutions in the search space and calculate the objective function of its present location. In search of better position the velocities are being exchanged between particles [12]. Suppose each particle $\mathrm{j}$ has its own position and velocity and $\mathrm{D}$ is the dimension of solution space. Firstly, the velocity and position of each particle is randomly generated in search space with some fitness function. With each iteration the position and velocity of each particle $\mathrm{j}$ on dimension $\mathrm{D}$ in search space with best fitness function is updated. PSO algorithm pseudo code is given below:

For every particle

Seteach particle $j$ with velocity in dimension $D$ with some fitness value $F V=F$

END

Do

For every particle

Compute its fitness function value

If new fitness value $F^{\prime}$ is better than previous fitness value $F$ then set new fitness value $F$ ' as new fitness value $F V=F$.
Now choose the particle $j$ with best FV from all particles as global best as $G B$

For every particle

Compute particle velocity bestowing to equation

Compute particle position bestowing to equation.

END

\section{MOTH FLAME OPTIMIZATION}

Moth flame Optimization is one of the Population based algorithm in which moths and flames both are solutions. Both moths and flames update theirs values in different ways. The moths are real exploration agents which move around the postulate search space and flames are the best position that is to be obtained with every iteration. Exploration and exploitation is inversely proportional to each other [11]. To maintain the balance between both for global optimization we will use population based algorithms. The main motivation of Moth Flame optimizer (MFO) is the triangulation process of moths in nature called transverse orientation. Moths are flying with respect to the moon with fixed angle. These fancy insects are stuck in impractical twisted trail near the artificial light. This algorithm gives very robust and optimum results in unnatural and unidentified search spaces. The pseudo code of the MFO algorithm is presented below:

Calculate the number of flames (flame numbers $=\mathrm{FN}$ ) Initialize the population of moths

Calculate the objective values $(\mathrm{OB})$ according to the equation For all moths

For all parameters
Update $r$ (convergence constant)
and $t$ ( parameter in spiral equation)
Calculate distance between moth
and flame $(D)$
Update the matrix $(M)$ of moth
w.r.t. corresponding moth
End
the updated objective value $\left(O B^{\prime}\right)$ with

Calculate the updated objective value (OB') with new $r, t, D$ and $M$.

Update the FN

End 


\section{RESULT AND SIMULATION:}

The simulation of the defined objective is achieved by using MATLAB 2014b software. The name MATLAB is stand for Matrix Laboratory which originally used for matrix computation. It is an interactive system; high performance language which does not required dimensioning due to use of array elements. In MATLAB program numbers of input fed likes random data signal, symbol size, modulation size and type, carriers, pilot, filters, noise, demodulator etc. and output of the system is shown in graphical form. The graphical output gives the flexibility to analyses, observe and draw the conclusion. The simulation parameters for the proposed system are given in table 1 .

\begin{tabular}{|l|l|}
\hline Parameter & Value \\
\hline No. of Subcarrier & 128 \\
\hline No. of pilot & 52 \\
\hline Channel length & 56 \\
\hline Modulation type & QAM \\
\hline No. of iteration & 80 \\
\hline
\end{tabular}

In this section, we will analyses and observe the result of the proposed technique. Figure 2 gives the OFDM signal before the transmission.

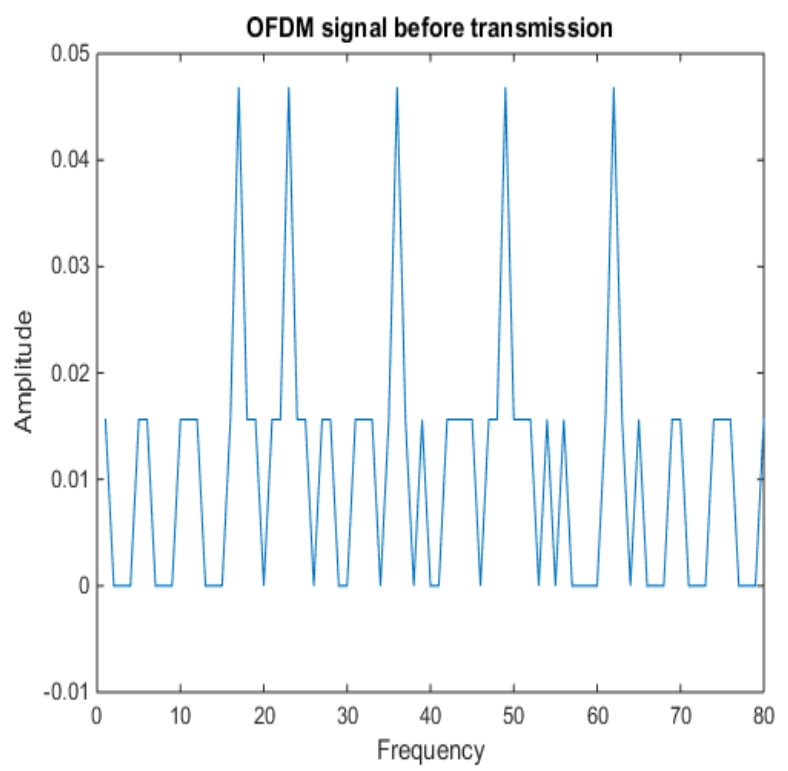

Figure 2: OFDM signal before the transmission

Figure 3 results the proposed Hybrid (LS+RLS) technique is the observed with the conventional Least Square (LS) technique. It is observed that with increase of SNR, bit error rate (BER) decreases as compare to LS approach. The performance evaluation comparison shows that the BER performance of the proposed hybrid technique is slightly better than the Least square technique. In other words, the proposed technique provides greater transmission rate and low execution time delay while slightly improving the BER performance.

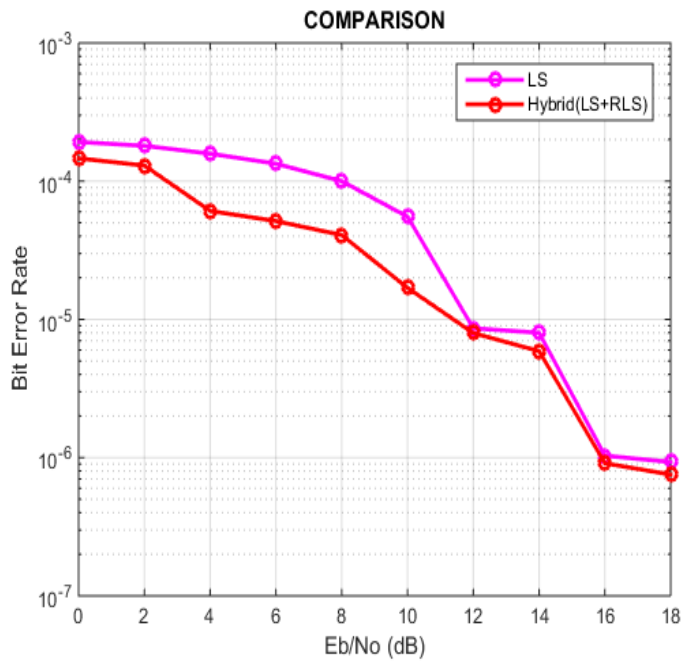

Figure 3: Comparison of LS and Hybrid (LS +RLS) technique

Figure 4 gives the BER performance of with PSO and Hybrid optimization (PSO+MFO) techniques. The hybrid optimizations give the better performance with increase in $\mathrm{Eb} / \mathrm{No}$.

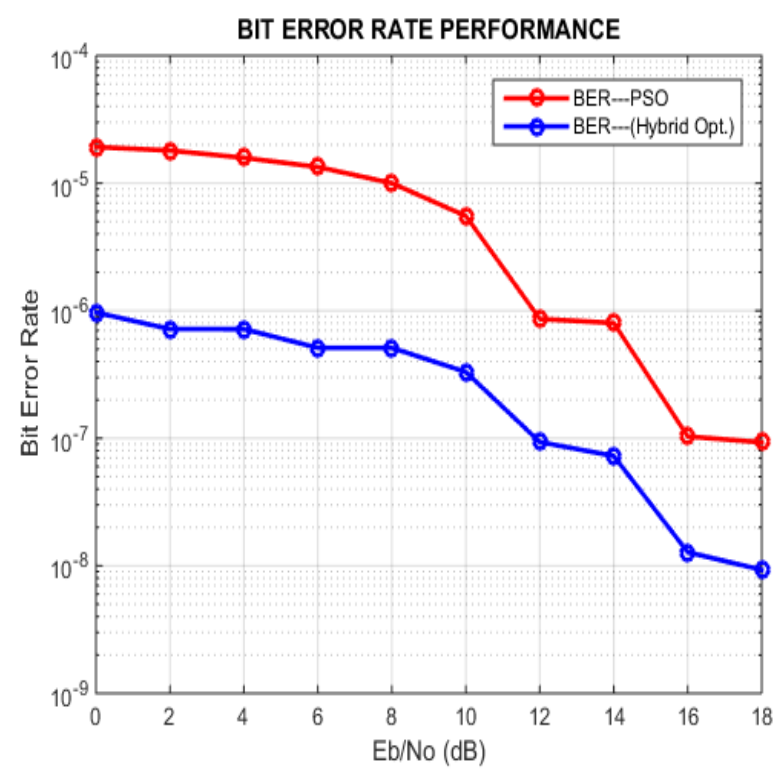

Figure 4: BER performance with PSO and Hybrid optimization (PSO+MFO)

Figure 5 represents the comparison of BER performance of LS, LS+RLS, PSO and PSO+MFO(hybrid) optimization techniques. 


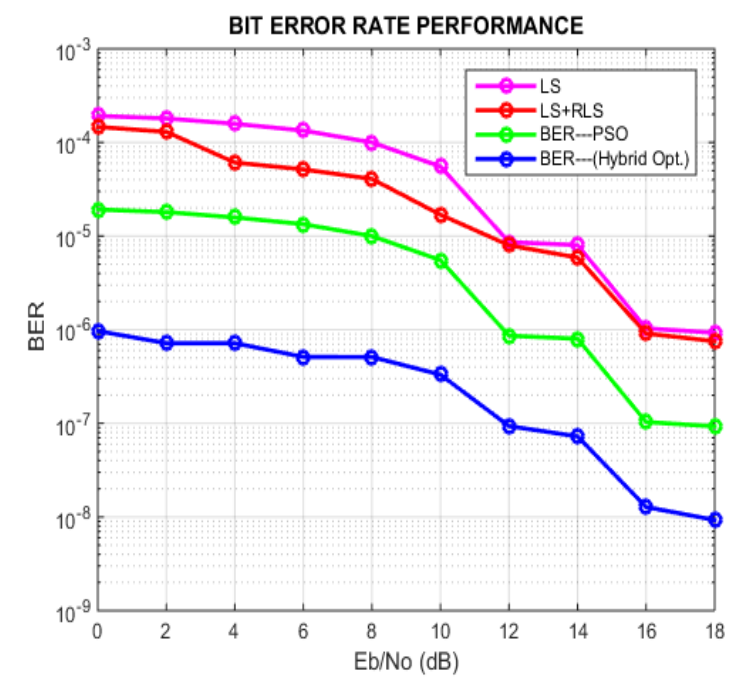

Figure 5: Comparison of BER performance of LS, LS+RLS, PSO and PSO+MFO (hybrid) optimization techniques

\section{CONCLUSION}

In this paper, we proposed and implemented bit error level reduction in channel estimation based OFDM system. Firstly, ofdm based system techniques with pilot aided synchronization and channel estimation is proposed using LS and Hybrid (LS+RLS) technique. It is used for the reduction and analysis of overhead interm of complexity, execution delay and computational complexity. Secondly, on proposed system is we apply optimization technique to get better results. The results of PSO and hybrid (PSO+MFO) optimization techniques give far better results. The graphs show that channel estimation based on pseudo-pilot with hybrid optimization gives optimum results. It has also been observed that system execution time and overhead reduced, transmission rate increases and BER performance is better than conventional OFDM system based channel estimation technique.

\section{REFERENCES}

[1] Ye Li, "Simplified channel estimation for OFDM systems with multiple transmit antennas", IEEE Transactions on Wireless Communications, vol. 1, no. 1, pp. 67-75, 2002.

[2] Y. Ma, "Pseudo-Pilot: A Novel Paradigm of Channel Estimation", IEEE Signal Processing Letters, vol. 23, no. 6, pp. 814-818, 2016

[3] T. S. Rappaport, Wireless Communications, Principles and Practice 2nd ed., Pearson Edu., vol.-1, pp. 356-376, 2002.

[4] D. Qu , S. Lu and T. Jiang, "Multi-block joint optimization for the peak-to-average power ratio reduction of FBMC-OQAM signals", IEEE Trans. Signal Process., vol. 61 , no. 7, pp.1605-1613, 2013.

[5] S.Venkatachalam,T.Manigandan, "Optimization of OFDM Systems Using Genetic Algorithm in FPGA," - International Journal of Computing Science and Communication Technologies, VOL.5 NO. 2, PP-854-859, Jan. 2013

[6] P. Aggarwal and V. A. Bohara, "A Nonlinear Downlink Multiuser MIMO-OFDM Systems," in IEEE Wireless Communications Letters, vol. 6, no. 3, pp. 414-417, June 2017.

[7] T. J. Lee and Y. C. Ko, "Channel Estimation and Data Detection in the Presence of Phase Noise in MIMO-OFDM Systems With Independent Oscillators," in IEEE Access, vol. 5, pp. 9647-9662, 2017.

[8] E. Eraslan and B. Daneshrad, "Low-Complexity Link Adaptation for Energy Efficiency Maximization in MIMO-OFDM Systems," in IEEE Transactions on Wireless Communications, vol. 16, no. 8, pp. 5102-5114, Aug. 2017.

[9] R. Kaur and . Mittal, "Implementation of neural network for channel estimation in OFDM network," 2nd International Conference on Contemporary Computing and Informatics (IC3I), 2016.

[10] Şimşir and N. Taşpınar, " hannel estimation using neural network in Orthogonal Frequency Division Multiplexing-Interleave Division Multiple Access (OFDM-IDMA) system," Telecommunications Symposium (ITS), 2014 International, Sao Paulo, pp. 1-5 2014.

[11] S. Mirjalili, "Moth-Flame Optimization Algorithm: A Novel Nature-inspired Heuristic Paradigm" , Knowledge-Based Systems (2015), doi: http://dx.doi.org/10.1016/j.knosys.2015.07.006" .

[12] L. Zajmi, F. Ahmed and A. Jaharadak, "Concepts, Methods, and Performances of Particle Swarm Optimization, Backpropagation, and Neural Networks", Applied Computational Intelligence and Soft Computing, vol. 2018, pp. 1-7, 2018. Available: $10.1155 / 2018 / 9547212$.

\section{AUTHORS PROFILE}

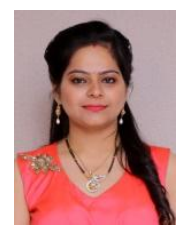

Mamta Arora graduated in Electronics and Communication Engineeringfrom RIMT-MAEC mandigobindgarh. I am completed Masters in Technology, Communication System from Electronics Department of Guru Nanak Dev. University, Amritsar. I am pursuing Ph.D in Electronics \& Communication Engineering from Chandigarh University, Mohali. I had also published more than 10 research papers in various international and national journals and conferences. His research interests include Wireless Communication, OFDM, 4G and soft computing.

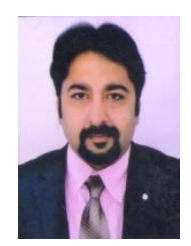

Paras Chawla received his B.Tech. (Honors), M.Tech. and Ph.D. degree in Electronics and Communication Engineering from Kurukshetra University, NIT, Kurukshetraand Thapar University, Patiala. He has 15 years of teaching experience and currently working as Professor \& HOD in ECE Department at Chandigarh University, Mohali (India). Total twenty M.Tech. Dissertations of various fields of ECE guided successfully. He is also guiding 07 students of $\mathrm{PhD}$. He received the "Coventor Scholarship award" from MANCEF, New Mexico-USA for his proposal titled "Performance \& Analysis of RF Front Section of Mobile Terminal Using RF MEMS DC Contact Switches", under the name of conference "Commercialization of Micro-Nano Systems Conference (COMS 2010)". His team received consecutive two years "Tenderfoot Award" collaborated given by American Astronautical Society, American Institute of Aeronautics, NASA/ Goddard Space Flight Centre, NASA/Jet Propulsion Laboratory \& Naval Research Laboratory for "CanSat Competition", June 2015 \& 2016, Burkett, Texas, USA.. 